\title{
ANÁLISE DE VIABILIADE TÉCNICA E ECONÔMICA DO EMPREGO GRANALHA DE GUSA NO FEA*
}

\author{
Tiago Seixas Bittencourt ${ }^{1}$ \\ Esdras Melo da Silva ${ }^{2}$ \\ João Paulo Carvalho Osório ${ }^{3}$ \\ Fernando de Souza Candido ${ }^{4}$
}

\section{Resumo}

A carga metálica do Forno Elétrico a Arco (FEA) representa bem mais que a matéria prima da rota semi integrada, representa o ponto de equilíbrio financeiro de uma operação siderúrgica sustentável. O fiel da balança econômica de uma operação baseada em um FEA baseia-se no custo do emprego metálico. Deste modo é razoável pensar que, escolher a combinação de sucatas que irá compor o mix de metálicos que abastecerá o FEA é uma tarefa que vai além das limitações técnicas do fenômeno de fusão e refino primário no referido reator. O emprego de resíduos metálicos diversos representa uma medida de sustentabilidade para a indústria siderúrgica, tanto sob a ótica ambiental, dado o reaproveitamento do resíduo, como na perspectiva econômica, haja vista a redução substancial nos custos de transformação. Testes realizados no FEA da CSN UPV possibilitaram avaliar os aspectos positivos e negativos do emprego de um resíduo metálico descartado durante a produção de cimentos. Os resultados encontrados demostram que, sob a perspectiva técnica, os aspectos positivos sobressaem aos negativos e, portanto, $O$ FEA representa uma ótima alternativa para o consumo sustentável do referido resíduo. Uma avaliação econômica aponta uma redução de custo do emprego metálico da ordem de $17 \%$.

Palavras-chave: Resíduos metálicos; FEA; Sustentabilidade.

\section{TECHNICAL AND ECONOMIC ANALYSIS ABOUT USE OF PIG IRON RESIDUALS AT EAF}

\section{Abstract}

The metallic charge used at Electric Arc Furnace (EAF) is much more than the raw material of semi integrated route, it even represents the financially breakeven point in a sustainable steelmaking operations. The true economic balance of an operation based on an EAF based on the scrap cost. Thus it is reasonable to think that, choose the scraps combination that will make up the best mix to be charged at the EAF is a task that goes beyond the technical limitations of the melting and primary refining phenomenon's in that reactor. The use of metallic residuals is a sustainability measure for the steel industry, both from the environmental viewpoint, since the reuse of waste, as the economic outlook, given the substantial reduction in processing costs. Tests got at UPV EAF evaluated the positives and negatives aspects of use of metallic residuals discarded during the production of cement. The results show that, under the technical perspective, the positives outnumber the negatives and therefore EAF is a great alternative for sustainable consumption that residue. An economic evaluation indicates a reduction of scrap cost about $17 \%$.

Keywords: Metalic residuals; EAF; Sustentability.

Engenheiro Especialista, Companhia Siderúrgica Nacional (CSN), Volta Redonda, RJ, Brasil.

2 Supervisor de Pátio de Sucata, Companhia Siderúrgica Nacional (CSN), Volta Redonda, RJ, Brasil.

3 Técnico de Desenvolvimento, Companhia Siderúrgica Nacional (CSN), Volta Redonda, RJ, Brasil.

4 Gerente Geral de Produção de Aços Longos, Companhia Siderúrgica Nacional (CSN), Volta

Redonda, RJ, Brasil. 


\section{INTRODUÇÃO}

A crescente ofensiva da produção de aço via rota semi integrada, aliado ao complexo modelo de recirculação de sucata ferrosa no mercado de metálicos, vem despertando interesse particular de engenheiros e pesquisadores do mundo todo, a respeito dos mecanismos envolvidos na operação de fusão e refino em Fornos Elétricos a Arco (FEA).

A prática de reciclagem de material metálico, além de ambientalmente correta, traz benefícios econômicos expressivos para a organização. Por outro lado o a disparidade entre o incremento dos volumes de produção de aço e a disposição dos bens de consumo para a reciclagem, cria uma dicotomia no mercado de metálicos, além de uma instabilidade na relação de oferta e demanda de sucata para a produção de aço via rota semi integrada, fazendo com que, cada vez mais se investigue materiais alternativos para o emprego no Forno Elétrico a Arco (FEA) para a produção de aço na rota semi integrada.

O presente trabalho propõe uma avaliação técnica, ambiental e econômica acerca do emprego de um resíduo metálico proveniente da fabricação de cimentos, doravante chamado de granalha de gusa, como matéria prima no FEA da CSN. A granalha de gusa é caracterizada como um resíduo da fabricação de cimentos, com granulometria variando de $0-5 \mathrm{~mm}$, com características químicas similares ao ferro gusa, metálico comumente empregado em fornos elétricos em todo o mundo.

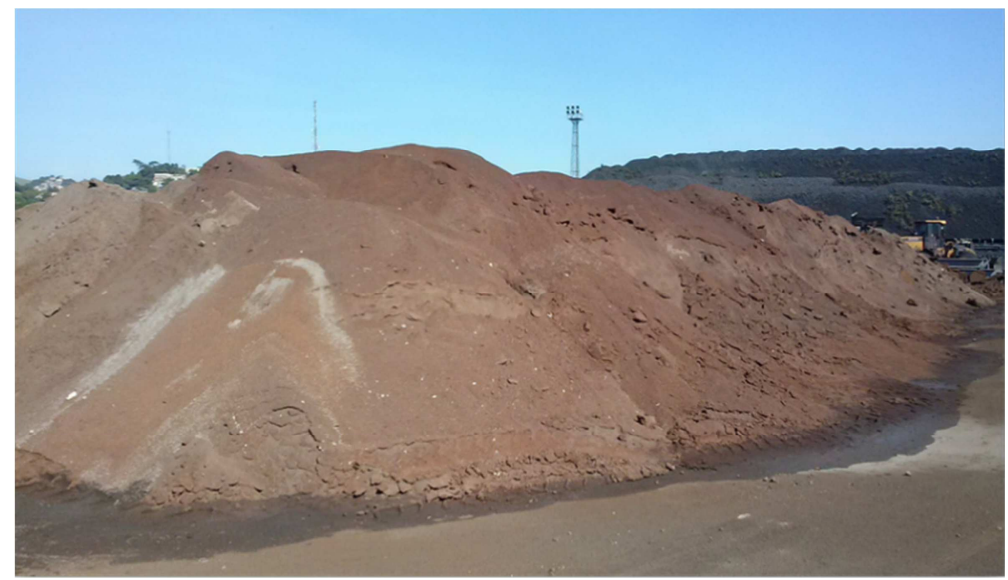

Figura 1. Pátio onde é estocada a granalha de gusa.

A com posição química da granalha de gusa é muito similar ao do ferro gusa, como pode ser visto na tabela 1 . Sendo seu percentual metálico praticamente idêntico, com um teor de carbono reduzido menor que $1 \%$ e silício variando de $0,2 \%$, aproximadamente. Teve-se notar o teor de enxofre do insumo, que é menor se comparado com o do ferro gusa, está sendo uma vantagem em relação a esse, mas sendo contrabalanceada pelo teor de fósforo um pouco mais elevado para a granalha. 
Tabela 1. Análise química comparativa entre a granalha de gusa e ferro gusa. Feita via espectroscopia de emissão atômica com plasma acoplado indutivamente.

\begin{tabular}{c|c|c|c}
\hline Parâmetro & u.m & Granalha & Gusa \\
\hline C & $\%$ & 3,81 & 4,5 \\
\hline Fe-total & $\%$ & 88,91 & 89 \\
\hline Si & $\%$ & 0,722 & 0,50 \\
\hline $\mathrm{Mn}$ & $\%$ & 0,430 & 0,60 \\
\hline $\mathrm{S}$ & $\%$ & 0,002 & 0,065 \\
\hline $\mathrm{P}$ & $\%$ & 0,181 & 0,100 \\
\hline
\end{tabular}

A origem da granalha de gusa vem do processo de fabricação de cimentos, onde a fração metálica da escória do alto forno que abastece a fábrica de cimentos é descartada durante o processo de moagem. Pode-se observar então que o insumo não era utilizado anteriormente, sendo estocado em pátios, agregando custo de estoque. Seu uso como carga metálica em carro torpedo já foi estudado, mas foi abortado devido a razões de segurança operacional, devido a projeção para fora do carro torpedo de material.

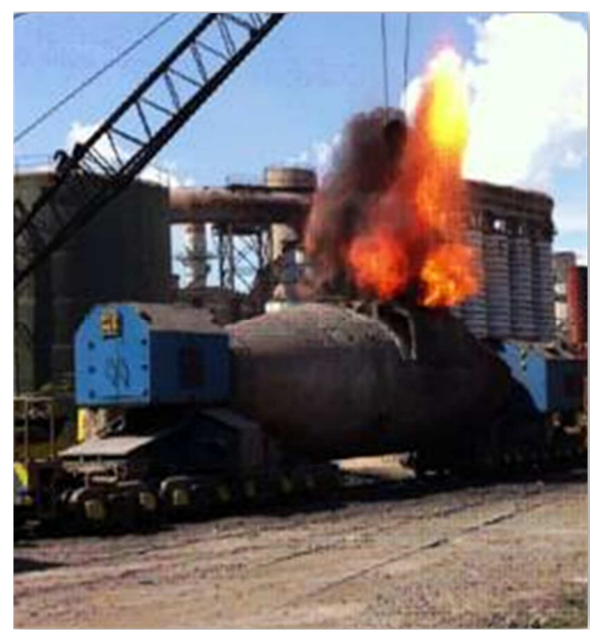

Figura 2. Teste feito com granalha sendo utilizada como carga metálica em carro torpedo. Abortado devido à projeção para fora do torpedo.

\subsection{Potencial Energético da Carga Metálica}

No FEA existem dois tipos diferentes de energia: química e elétrica. A energia elétrica é proveniente do sistema elétrico de potência do FEA, enquanto que a energia química é obtida através de reações que ocorrem no forno. O balanço de energia é terminado pela soma da energia elétrica adicionada através dos eletrodos mais a energia química proveniente das reações exotérmicas, sendo descontada a parcela de energia endotérmica, sendo essa soma igualada a energia necessária para fundir a sucata fria que é carregada no FEA.

\subsubsection{Energia química}

Uma reação química indica um rearranjo de elétrons e núcleos de uma estrutura, isso ocorre com absorção (endotérmicas) ou liberação (exotérmicas) de calor. No caso do FEA ocorrem ambos os tipos de reações, no entanto serão apresentadas apenas as exotérmicas, ou seja, as que fornecem energia ao sistema. 


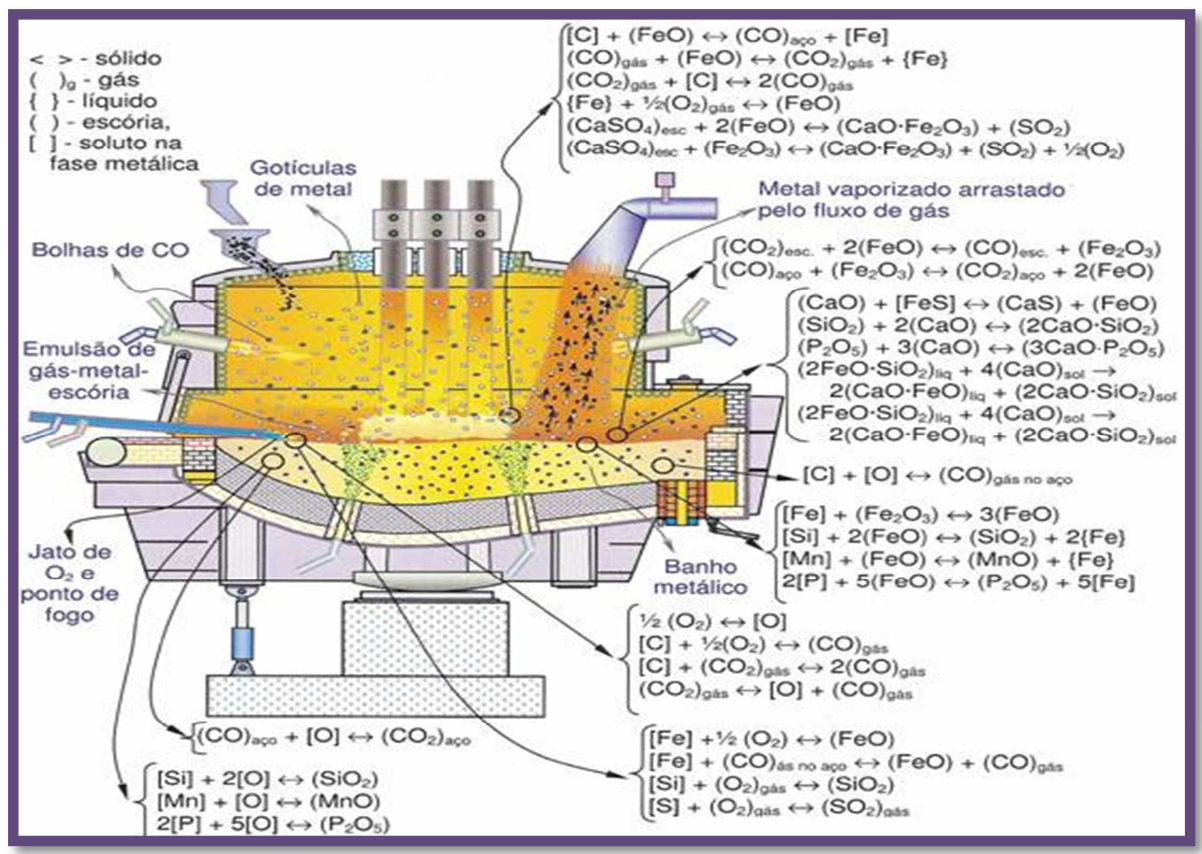

Figura 3. Esquema da localização das reações que ocorrem em um FEA.

Além da energia proveniente das reações químicas, também há à combustão do gás natural proveniente dos queimadores.

\subsubsection{Geração de energia química no FEA}

Vários elementos estão presentes tanto na sucata quanto no gusa, e esses elementos ao reagirem com oxigênio, fornecem energia para o forno de acordo com as reações apresentadas a baixo.

$\mathrm{Si}+\mathrm{O}_{2} \rightarrow \mathrm{SiO}_{2}+$ geração de calor

$\mathrm{Mn}+1 / 2 \mathrm{O}_{2} \rightarrow \mathrm{MnO}+$ geração de calor

$\mathrm{C}+1 / 2 \mathrm{O}_{2} \rightarrow \mathrm{CO}^{\pi}+$ geração de calor

$\mathrm{CO}+1 / 2 \mathrm{O}_{2} \rightarrow \mathrm{CO}^{\pi}+$ geração de calor

$\mathrm{Fe}+1 / 2 \mathrm{O}_{2} \rightarrow \mathrm{FeO}+$ geração de calor

A energia gerada pelas reações é explicada pelo fenômeno conhecido como entalpia. As substâncias possuem uma entalpia de formação, ou seja, calor gerado na formação ou fornecido para que a reação aconteça. Como resultado a temperatura global do sistema aumenta no caso de reações exotérmicas ou diminui no caso de reações endotérmicas.

\subsubsection{Energia elétrica}

O cálculo da energia elétrica necessária para o balanço térmico de um FEA é calculada em função das outras energias, tanto de perdas como de geradas. A energia gerada será a energia química e as energias perdidas contabilizam energia necessária para fundir a carga, perdas para fusão e aquecimento de escória, perdas no circuito secundário do forno, perdas nos painéis refrigerados e outras perdas, provenientes do despoeiramento, aberturas, etc. 


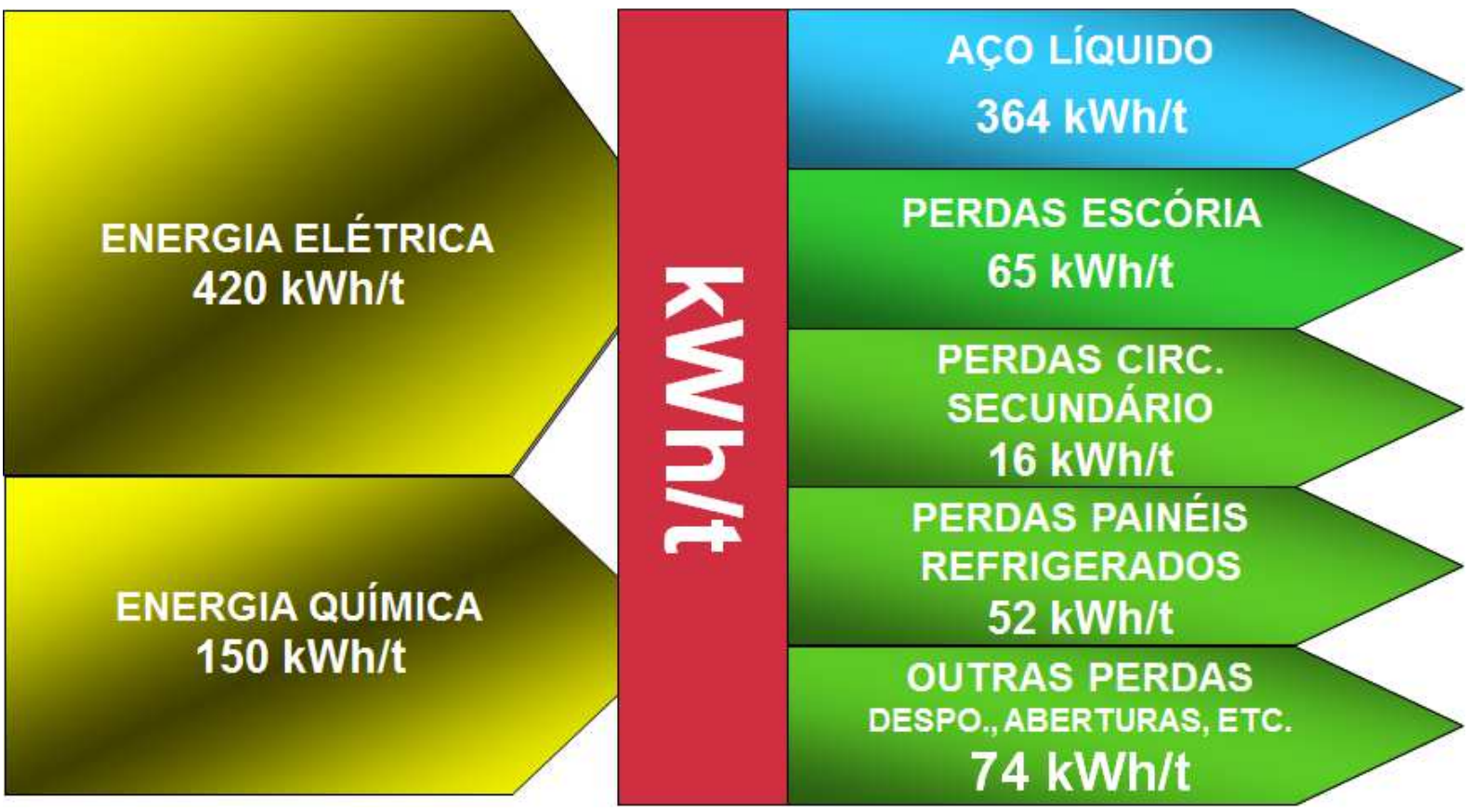

Figura 4. Exemplo de balanço de energia em um FEA

\subsection{Termodinâmica da Fusão de Sucata}

A termodinâmica de fusão leva em consideração a formula de energia necessária para elevar determinado material a determinada temperatura e estado físico, dada por:

$E_{\text {SUCATA }}=C_{1} \cdot m \cdot\left(t_{f}-t_{i}\right)+C_{2} \cdot m ;$

Onde:

$E_{\text {SUCATA }}=$ energia necessária para elevação da temperatura e/ou transformação de fase;

$\mathrm{C}_{1}=$ calor especifico a pressão constate do material no estado sólido;

$\mathrm{C}_{2}=$ calor sensível do aço do estado sólido para o estado líquido;

$\mathrm{m}=$ massa, em toneladas;

$\mathrm{t}_{\mathrm{f}}=$ temperatura final;

$\mathrm{t}_{\mathrm{i}}=$ temperatura inicial.

A teoria explica que para fundir certa massa de sucata, temos que fornecer energia para que elevar sua temperatura até a temperatura de fusão, aquecê-la para concretizar transformação de fase e após aquecê-la até a temperatura de vazamento adequada.

Tabela 2. Esquema da termodinâmica de fusão da sucata.

Aquecimento Transformação

até a

temperatura de

fusão. da fase sólida para a fase líquida.

$\longrightarrow \begin{aligned} & \text { Aquecimento } \\ & \text { até a } \\ & \text { temperatura de } \\ & \text { vazamento. }\end{aligned}$

Baseada no calor específico médio do aço, seu calor latente de fusão, em termos de aplicações elétricas, tem o valor de $C_{1}=0,194 \mathrm{kWh} / \mathrm{t}$, até a temperatura de $1538^{\circ} \mathrm{C}$. Atingida a temperatura de fusão, o aço inicia sua transformação de fase da fase sólida para a líquida, tendo um calor sensível de $\mathrm{C}_{2}=58 \mathrm{kWh} / \mathrm{t}$. Completada tal 
transformação, o aço permanecerá na temperatura de fusão, sendo necessário aquecê-lo até a temperatura de vazamento, e como o aço encontra-se no estado líquido, seu calor latente varia, para o valor de $\mathrm{C}_{3}=0,223 \mathrm{kWh} / \mathrm{t}$. Então a equação torna-se.

$E_{\text {SUCATA }}=C_{1} \cdot m \cdot\left(t_{\text {fus }}-t_{\text {ini }}\right)+C_{2} \cdot m+C_{3} \cdot m \cdot\left(t_{\text {vaz }}-t_{\text {fus }}\right)$

\section{MATERIAL E MÉTODOS}

Primeiramente foi usada a metodologia PDCA com o plano de ação 5W1H acoplado, onde foram contempladas as principais pendencias a serem tratadas. As ações que foram definidas:

- Definir quantidade de material para testes.

- Operacionalizar o transporte de material para teste no FEA.

- Acompanhar testes do material no FEA.

- Elaborar relatório técnico sobre os testes.

- Padronizar o emprego de granalha de gusa.

O escopo levantado para os testes, levando em conta os aspectos de balanço de massa, segurança operacional, balanço de energia e custo do emprego foi calculado é apresentado na tabela 3.

Tabela 3. Escopo da quantidade de material usado nos testes.

\begin{tabular}{c|c|c|c}
\hline CORRIDA & $\begin{array}{c}\text { Gusa } \\
\text { original }\end{array}$ & $\begin{array}{c}\text { Gusa } \\
\text { teste }\end{array}$ & $\begin{array}{c}\text { Granalha } \\
\text { teste }\end{array}$ \\
\hline A711 & 15 & 10,295 & 4,705 \\
\hline $\mathrm{A} 712$ & 15 & 12,129 & 2,871 \\
\hline $\mathrm{A} 713$ & 15 & 11,604 & 3,396 \\
\hline $\mathrm{A} 714$ & 15 & 13,019 & 1,981 \\
\hline $\mathrm{A} 715$ & 15 & 13,088 & 1,912 \\
\hline $\mathrm{A} 716$ & 15 & 12,771 & 2,229 \\
\hline $\mathrm{A} 717$ & 15 & 13,029 & 1,971 \\
\hline $\mathrm{A} 718$ & 15 & 13,103 & 1,897 \\
\hline $\mathrm{A} 719$ & 15 & 12,391 & 2,609 \\
\hline $\mathrm{A} 720$ & 15 & 12,531 & 2,469 \\
\hline A721 & 15 & 11,953 & 3,047 \\
\hline TOTAL & 165 & 135,913 & 29,087 \\
\hline
\end{tabular}

\section{RESULTADOS E DISCUSSÃO}

\subsection{Abertura do Canal EBT}

Durante os testes, uma das preocupações foi se ocorreria obstrução do canal EBT e como mostra a figura 5, esse problema só foi encontrado em 2 das 38 corridas analisadas com a adição de granalha, sendo os índices 1 para abertura livre e 0 para abertura forçada. A normalidade de qualquer aciaria é abertura livre em sua grande maioria das vezes. 


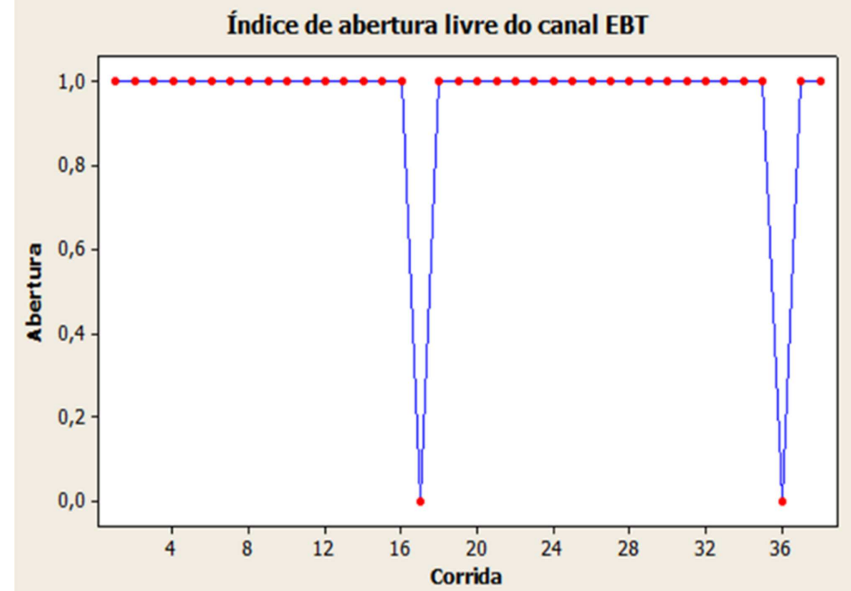

Figura 5. Análise dos resultados de abertura do EBT, 1 - abertura livre, 0 - abertura forçada.

\subsection{Composição das Corridas Teste}

Foram medidas as composições químicas dos aços produzidos usando granalha de gusa, para observar os elementos que variavam entre o gusa sólido e a granalha de gusa, pois poderia haver um rebote de algum desses elementos sendo eles, carbono, silício, enxofre e fósforo. O resultado das corridas analisadas podem ser observados nas figuras 6 a 9 .

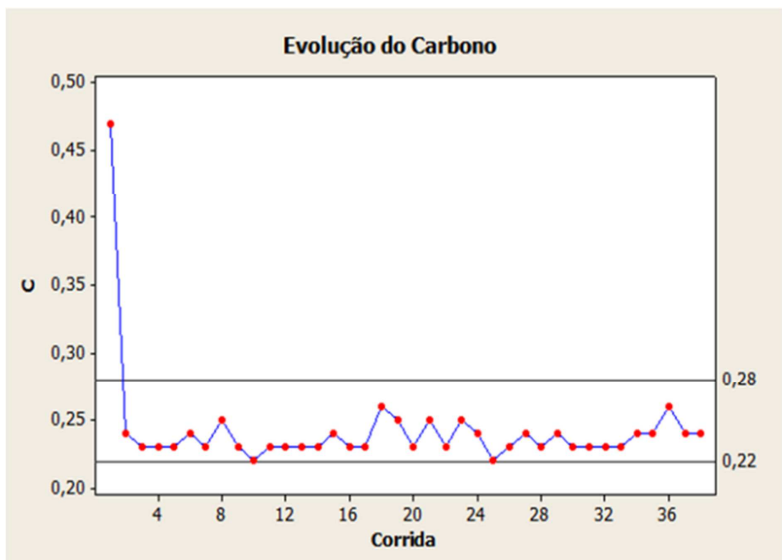

Figura 6. Variação do carbono durante as corridas teste.

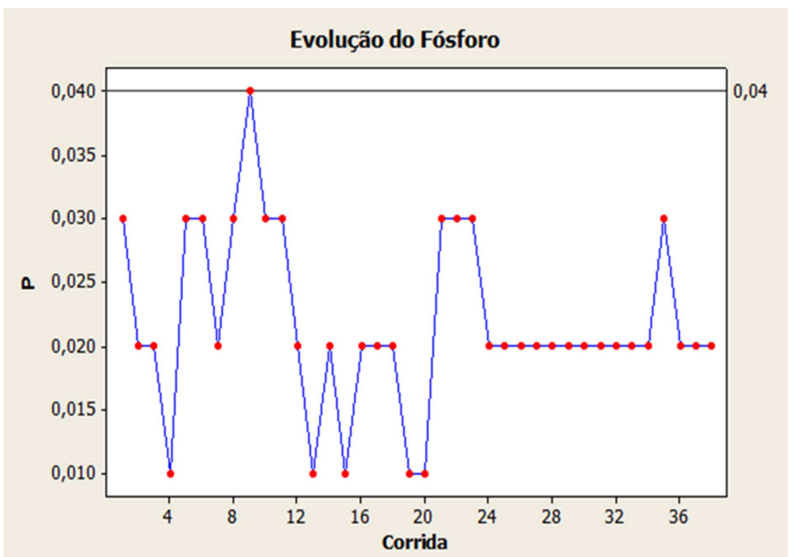

Figura 7. Variação do fósforo durante as corridas teste. 


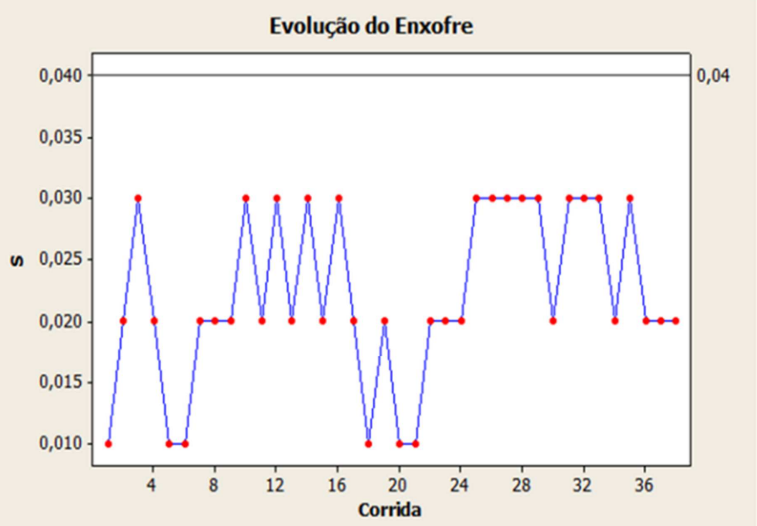

Figura 8. Variação do enxofre durante as corridas teste.

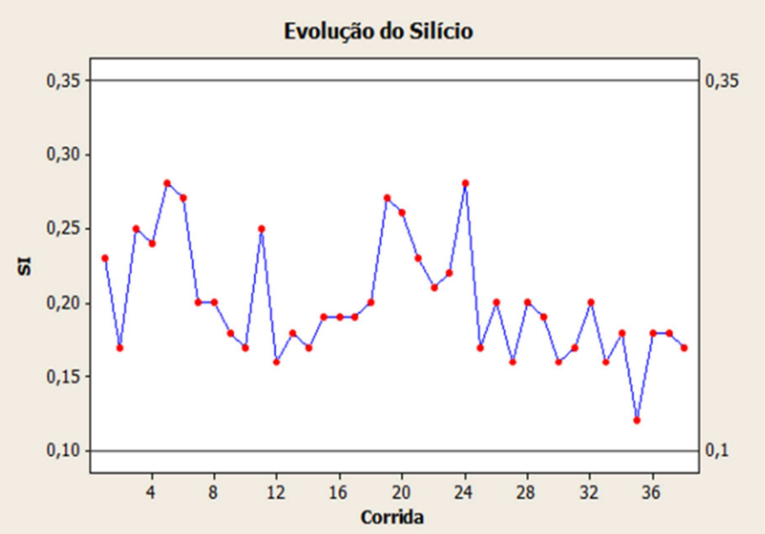

Figura 9. Variação do silício durante as corridas teste.

Portanto, não houveram grandes variações fora da faixa dos elementos onde o teor varia entre os insumos gusa sólido e granalha de gusa.

\section{CONCLUSÃO}

Não há impedimento relacionado à segurança operacional quanto ao emprego do referido material, haja visto que o material é empregado como carga sólida no FEA. O emprego do material não afetou a sanidade química do produto final da aciaria, mostrando-se, portanto tecnicamente viável (variação de rendimento de apenas $0,2 \%$ via Balanço de Massas). Empregar o material como carga metálica do FEA também se mostra uma substancial vantagem econômica.

\section{Agradecimentos}

Aos colaboradores que ajudaram incondicionalmente o projeto em suas fases de testes e aos que incentivaram e colaboraram de forma técnica, teoria e incentivadora.

\section{BIBLIOGRAFIA}

1 Bosi, Fernando, Ribeiro, Barros, Delmar. Energias, ABM - Curso Aciaria Elétrica. 